\title{
Plasmid DNA delivery using fluorescein-labeled arginine-rich peptides
}

Makoto Oba $^{\mathrm{a}, *}$, Yosuke Demizu ${ }^{\mathrm{b}}$, Hiroko Yamashita ${ }^{\mathrm{b}, \mathrm{c}}$, Masaaki Kurihara ${ }^{\mathrm{b}, \mathrm{c}}$, Masakazu Tanaka $^{\mathrm{a}}$

aGraduate School of Biomedical Sciences, Nagasaki University, Nagasaki 852-8521, Japan

bDivision of Organic Chemistry, National Institute of Health Sciences, Tokyo 158-8501, Japan

${ }^{c}$ Graduate School of Bioscience and Biotechnology, Tokyo Institute of Technology, Yokohama 226-8501, Japan

*To whom correspondence should be addressed:

Makoto Oba

Graduate School of Biomedical Sciences, Nagasaki University, Nagasaki 852-8521, Japan

Tel: +81-95-819-2424; Fax: +81-95-819-2424

E-mail: moba@nagasaki-u.ac.jp (M. Oba)

\section{Abstract}


Arginine (Arg)-rich peptides exhibit an effective cell-penetrating ability and deliver membrane-impermeable compounds into cells. In the present study, three types of Arg-rich peptides, R9 containing nine Arg residues, (RRG)3 containing six Arg and three glycine (Gly) residues, and (RRU)3 containing six Arg and three $\alpha$-aminoisobutyric acid (Aib) residues, were evaluated for their plasmid DNA (pDNA) delivery and cell-penetrating abilities. The transfection efficiency of R9/pDNA complexes was much higher than those of (RRG)3 and (RRU)3/pDNA complexes, and was derived from the enhanced cellular uptake of R9/pDNA complexes. The replacement of three Arg residues with the neutral amino acid Gly and hydrophobic amino acid Aib drastically changed the cell-penetrating ability and physicochemical properties of peptide/pDNA complexes, resulting in markedly reduced transfection efficiency. A comparison of the R9 peptide administration forms between a peptide alone and peptide/pDNA complex revealed that the uptake of R9 peptides was more efficient for the complex than the peptide alone, but occurred through the same internalization mechanism. The results of the present study will contribute to the design of novel Arg-rich cell-penetrating peptides for pDNA delivery.

\section{Keywords}

Cell-penetrating peptide; Arginine; Gene transfer; Drug delivery system

\section{Introduction}


Arginine (Arg)-rich peptides are well-known cell-penetrating peptides (CPPs) that can cross the plasma membrane in not only in vitro cultured cells, but also in vivo animal tissues [1-3]. In addition to Arg-rich peptides themselves, membrane-impermeable compounds can be delivered into cells by Arg-rich peptides. Therefore, previous studies focused on developing novel CPPs based on Arg-rich peptides including their derivatives and delivery systems for membrane-impermeable drugs, biological macromolecules, and nanosized materials [4-7]. Nucleic acids such as antisense DNA, plasmid DNA (pDNA), and siRNA are one of the major targets for delivery systems by Arg-rich peptides [8-10]. Electrostatic interactions between cationic guanidino groups in Arg-rich peptides and anionic phosphate groups in DNA or RNA afford polyion complexes, the physicochemical properties and transfection abilities of which depend on their peptide sequences, peptide lengths, and hydrophobic modifications [8-11].

In the present study, we examined pDNA delivery and the cell-penetrating abilities of fluorescein-labeled Arg-rich peptides. The peptides described herein were three types of nonapeptides composed of 6-carboxyfluorescein (6-FAM) as the fluorescent label, $\beta$-alanine ( $\beta$-Ala) as the linker, and nine amino acids: cationic L-Arg, neutral glycine (Gly), and/or hydrophobic $\alpha$-aminoisobutyric acid (Aib), 6-FAM- $\beta$-Ala-(L-Arg) ${ }_{9}-\mathrm{NH}_{2} \quad$ (R9), 6-FAM- $\beta$-Ala-(L-Arg-L-Arg-Gly) $)_{3}-\mathrm{NH}_{2} \quad$ [(RRG)3], and 6-FAM- $\beta$-Ala-(L-Arg-L-Arg-Aib) $)_{3}-\mathrm{NH}_{2}$ [(RRU)3] (Figure 1). The cell-penetrating abilities of peptides have generally been evaluated using peptides with fluorescent labels and gene delivery systems are developed using non-fluorescent labeled peptides; and 
therefore, these aspects have been achieved separately. In the present study, the same fluorescein-labeled peptides were used to evaluate the transfection and cell-penetrating abilities of peptide/pDNA complexes from the viewpoint of peptide behavior. By physicochemically evaluating peptide/pDNA complexes and performing in vitro assays of peptides and peptide/pDNA complexes against cultured cells, R9 peptides were found to possess the highest transfection and cell-penetrating abilities among the three types of peptides examined. R9 peptides were internalized into cells more efficiently for R9/pDNA complexes than for R9 peptides alone in spite of the same mechanism of internalization. These results will help in the design and development of Arg-rich peptides for CPPs and pDNA delivery.

\section{Materials and Methods}

\section{2-1. Materials}

Dullbecco's modified Eagle’s medium (DMEM) was obtained from Sigma-Aldrich Co. (St. Louis, MO). A micro BCA protein assay reagent kit was purchased from Pierce (Rockford, IL). The luciferase assay kit was a product of Promega (Madison, WI). Plasmid pCAcc+Luc, coding for firefly luciferase under the control of the CAG promoter, was provided by the RIKEN Gene Bank (Tsukuba, Japan), amplified in competent DH5a Escherichia coli, and then purified using a HiSpeed Plasmid MaxiKit purchased from Qiagen Sciences (Germantown, MD). Cell lysis buffer M was obtained from Wako Pure Chem. Co., Inc. (Osaka, Japan). Hoechst 33342 was purchased from 
Dojindo Laboratories (Kumamoto, Japan). LysoTracker Red was purchased from Molecular Probes (Eugene, OR).

\section{2-2. Peptide synthesis}

The

peptides

6-FAM- $\beta$-Ala-(L-Arg) ${ }_{9}-\mathrm{NH}_{2}$

(R9),

6-FAM- $\beta$-Ala-(L-Arg-L-Arg-Gly) ${ }_{3}-\mathrm{NH}_{2}$

[(RRG)3],

and

6-FAM- $\beta$-Ala-(L-Arg-L-Arg-Aib) $)_{3}-\mathrm{NH}_{2} \quad$ [(RRU)3] were synthesized as previously reported (Figure 1) [12]. The crude peptides were purified by reversed-phase HPLC. The purified peptides were characterized by analytical reversed-phase HPLC and matrix-assisted laser desorption/ionization mass spectrometry.

\section{2-3. Preparation of peptide/pDNA complexes}

Each peptide and pDNA was dissolved separately in $10 \mathrm{mM}$ Hepes buffer (pH 7.3). A two-fold excess volume of peptide solutions of various concentrations was added to the pDNA solution to form peptide/pDNA complexes with different compositions. The final pDNA concentration was adjusted to $33.3 \mu \mathrm{g} / \mathrm{mL}$ and complex solutions were stored at room temperature for 15 min prior to use. The charge ratio was defined as the residual molar ratio of the guanidino groups of peptides to the phosphate groups of pDNA.

\section{2-4. Dynamic light scattering (DLS) measurements}


The sizes of the peptide/pDNA complexes were evaluated by DLS using Nano ZS (ZEN3600, Malvern Instruments, Ltd., UK). A He-Ne ion laser (633 nm) was used as the incident beam. Light scattering data were obtained at a detection angle of $173^{\circ}$ and a temperature of $25^{\circ} \mathrm{C}$ and were subsequently analyzed by the cumulant method to obtain the hydrodynamic diameters and polydispersity indices (PDI) $\left(\mu / \Gamma^{2}\right)$ of the complexes. Results were presented as the mean and standard deviation obtained from 3 measurements (Table 1).

\section{2-5. Zeta-potential measurements}

The zeta-potentials of peptide/pDNA complexes were evaluated by the laser-Doppler electrophoresis method using Nano ZS with a He-Ne ion laser (633 nm). Zeta-potential measurements were carried out at $25^{\circ} \mathrm{C}$. A scattering angle of $173^{\circ}$ was used in these measurements. Results were presented as the mean and standard deviation obtained from 3 measurements (Table 1).

\section{2-6. Transfection}

Huh-7 and HeLa cells were separately seeded onto 24-well culture plates (10,000 cells/well) and incubated overnight in $400 \mu \mathrm{L}$ of DMEM containing $10 \%$ fetal bovine serum (FBS). The medium was exchanged and the peptide/pDNA complex solutions (33.3 $\mu \mathrm{g} \mathrm{pDNA} / \mathrm{mL}$ ) prepared at various charge ratios, naked pDNA, or commercially available TurboFect/pDNA prepared according to the manufacturer's protocol were 
applied to each well. The amount of pDNA was adjusted to $1 \mu$ g per well. After a 24-h incubation, the medium was replaced with $400 \mu \mathrm{L}$ of fresh medium, followed by further incubations for the indicated times (post-incubation). Luciferase gene expression was then evaluated based on photoluminescence intensity using the luciferase assay kit and a luminometer (Gene Light GL-210A, Microtec. Co., Ltd., Chiba, Japan). The amount of protein in each well was concomitantly determined using a Micro BCA protein assay kit. Results were presented as the mean and standard deviation obtained from 4 samples (Figures 2 and 3).

\section{2-7. Cellular uptake}

Huh-7 and HeLa cells were separately seeded onto 24-well culture plates (10,000 cells/well) and incubated overnight in $400 \mu \mathrm{L}$ of DMEM containing 10\% FBS. The medium was exchanged and the peptide or peptide/pDNA complex solutions (33.3 $\mu \mathrm{g}$ pDNA/mL) prepared at various charge ratios were applied to each well at an appropriate concentration. The amount of pDNA was adjusted to $1 \mu \mathrm{g}$ per well. The concentration of the guanidino groups in the medium treated with peptide/pDNA complexes prepared at a charge ratio $=2$ was $15.4 \mu \mathrm{M}$. The medium was removed after a 24-h incubation, and the cells were washed with ice-cold PBS and trypsinized. After the addition of medium containing $10 \%$ FBS, cells were centrifuged at 1,600 rpm for 3 min at $4^{\circ} \mathrm{C}$. The cell pellets obtained were suspended in ice-cold PBS, centrifuged at 1,600 rpm for $3 \mathrm{~min}$ at $4^{\circ} \mathrm{C}$, and treated with Cell lysis buffer M. The fluorescence 
intensity of each lysate was measured using a spectrofluorometer (ND-3300, NanoDrop, Wilmington, DE). The amount of protein in each well was concomitantly determined using a Micro BCA protein assay kit. Results were presented as the mean and standard deviation obtained from 3 samples (Figure 4).

\section{2-8. Cell viability}

Huh-7 and HeLa cells were separately seeded onto 96-well culture plates (2,500 cells/well) and incubated in $100 \mu \mathrm{L}$ of DMEM containing $10 \%$ FBS. The medium was then replaced with fresh medium containing 10\% FBS, and the peptide or peptide/pDNA complex solutions were added to each well at the appropriate concentration. The amount of pDNA was adjusted to $0.25 \mu \mathrm{g}$ per well. The concentration of guanidino groups in the medium treated with peptide/pDNA complexes prepared at a charge ratio = 2 was $15.4 \mu \mathrm{M}$. Cell counting kit-8 was used after a 24-h incubation according to the manufacturer's protocol. Cell viability was evaluated on the basis of the absorbance of formazan from each well, where $100 \%$ cell viability was calculated from the wells without peptides. Results were presented as the mean and standard deviation obtained from 6 samples (Figure 5).

\section{2-9. Fluorescence measurements}

The fluorescence intensities of peptide solutions prepared at various concentrations and peptide/pDNA complex solutions prepared at various charge ratios were measured 
using a spectrofluorometer (ND-3300). The concentration of guanidino groups in the peptide/pDNA complex solution $(33.3 \mu \mathrm{g} \mathrm{pDNA} / \mathrm{mL})$ prepared at a charge ratio $=1$ was 102.6 $\mu \mathrm{M}$. Results were presented as the mean and standard deviation obtained from 3 measurements (Figure 6).

\section{2-10. Inhibition of endocytosis}

Huh-7 cells were seeded onto 24-well culture plates (40,000 cells/well) and incubated in $400 \mu \mathrm{L}$ of DMEM containing 10\% FBS. After the replacement of medium with fresh medium containing 10\% FBS in the absence or presence of amiloride (5 mM), chlorpromazine $(10 \mu \mathrm{g} / \mathrm{mL})$, or nystatin $(25 \mu \mathrm{g} / \mathrm{mL})$, cells were pre-incubated at $37^{\circ} \mathrm{C}$ or $4^{\circ} \mathrm{C}$ for $10 \mathrm{~min}$ (for amiloride) or $30 \mathrm{~min}$ (for others). $\mathrm{R} 9$ peptide or $\mathrm{R} 9 / \mathrm{pDNA}$ complex (charge ratio $=8$ ) solutions were applied to each well at a guanidino group concentration of $61.5 \mu \mathrm{M}$ and $1 \mu \mathrm{g}$ pDNA. After a 2-h incubation at $37^{\circ} \mathrm{C}$ or $4^{\circ} \mathrm{C}$, the medium was removed, and the cells were washed with ice-cold PBS and trypsinized. After the addition of medium containing 10\% FBS, cells were centrifuged at 1,600 rpm for $3 \mathrm{~min}$ at $4^{\circ} \mathrm{C}$. The cell pellets obtained were suspended in ice-cold PBS, centrifuged at 1,600 rpm for $3 \mathrm{~min}$ at $4^{\circ} \mathrm{C}$, and then treated with Cell lysis buffer $\mathrm{M}$. The fluorescence intensity of each lysate was measured using a spectrofluorometer (ND-3300). The amount of protein in each well was concomitantly determined using the BCA protein assay reagent kit. Results were presented as the mean and standard deviation obtained from 3 samples (Figure 7). 


\section{2-11. Confocal laser scanning microscope (CLSM) observations}

Huh-7 cells were seeded onto 8-well chambered cover glasses (Iwaki, Tokyo, Japan) (20,000 cells/well) and incubated overnight in $200 \mu \mathrm{L}$ of DMEM containing $10 \%$ FBS. The medium was then replaced with fresh medium containing 10\% FBS, and R9 peptide or R9/pDNA complex (charge ratio $=8$ ) solutions were applied to each well at a guanidino group concentration of $61.5 \mu \mathrm{M}$ and $0.5 \mu \mathrm{g}$ pDNA. After a 24-h incubation, the medium was removed, and the cells were washed 3 times with ice-cold PBS supplemented with heparin (20 units/mL). Intracellular distributions were observed by CLSM after staining late endosomes/lysosomes with LysoTracker Red and nuclei with Hoechst 33342. CLSM observations were performed using LSM 710 (Carl Zeiss, Oberlochen, Germany) with a Plan-Apochromat 63x/1.4 objective (Carl Zeiss) at an excitation wavelength of $405 \mathrm{~nm}$ (UV laser) for Hoechst 33342, $488 \mathrm{~nm}$ (Ar laser) for peptides, and $543 \mathrm{~nm}$ (He-Ne laser) for LysoTracker Red (Figure 8A). The rate of the colocalization of peptides with LysoTracker Red was quantified [13]. The colocalization ratio was quantified as follows:

Colocalization ratio $(\%)=$ peptide pixels colocalization $_{\text {peptide }}$ pixels potal $_{\text {x }} 100$

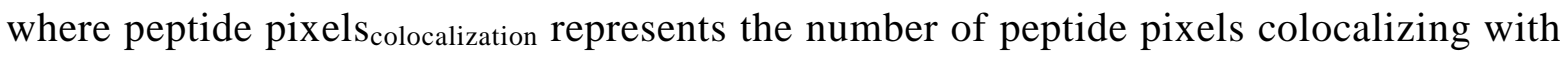
LysoTracker Red in the cell, and peptide pixelstotal represents the number of all the pixels in the cell. Results were represented as the mean and standard deviation obtained from 16 cells (Figure 8B). 


\section{Results}

\section{3-1. Transfection efficiencies of peptide/pDNA complexes}

The transfection efficiencies of the R9, (RRG)3, and (RRU)3/pDNA complexes prepared at charge ratios $=2,4$, and 8 were evaluated against Huh-7 and HeLa cells using the luciferase assay (Figure 2). The transfection efficiency of R9/pDNA complexes increased with the charge ratio against both cells, and reached a similar value to that of a commercially available TurboFect at a charge ratio $=8$ against Huh-7 cells. However, (RRG)3 and (RRU)3/pDNA complexes showed almost no transfection efficiencies, similar to naked pDNA, at all charge ratios against both cells.

\section{3-2. Effects of the post-incubation time on transfection efficiency}

The transfection experiments described in the preceding section were performed under the condition of a 24-h post-incubation after a 24-h incubation of peptides/pDNA complexes with the cultured cells (Figure 2). Additional transfection experiments were repeated against Huh-7 and HeLa cells at conditions with 24-, 48-, and 72-h post-incubations, in order to confirm the influence of post-incubation times on transfection efficiencies (Figure 3). The transfection efficiency of R9/pDNA complexes was maintained without marked decreases or increases at any charge ratio (Figures 3A and 3D). (RRG)3/pDNA complexes showed the maximum transfection efficiency at a charge ratio $=4$ after a 48 -h post-incubation against Huh-7 cells (Figure 
3B), while the maximum transfection efficiencies of (RRU)3/pDNA complexes at charge ratios $=4$ and 8 were after 48 -h and 72-h post-incubations, respectively (Figure 3C). After a 24-h post-incubation, almost no transfection efficiency was observed in Huh-7 cells treated with (RRG)3 and (RRU)3/pDNA complexes (Figure 2); however, appropriate post-incubation times led to the transfection of their pDNA (Figures 3B and 3C). (RRG)3 and (RRU)/pDNA complexes showed no transfection ability against HeLa cells at any charge ratio and post-incubation time (Figures 3E and 3F).

\section{3-3. Cellular uptake and cytotoxicity of peptides and peptide/pDNA complexes}

The cellular uptake of peptides added to peptide/pDNA complexes at charge ratios = 2, 4, and 8 , and that added to peptides alone without pDNA at appropriate concentrations corresponding to each charge ratio were evaluated in order to elucidate the mechanism underlying the markedly high transfection ability of R9 peptides (Figure 4). The cell culture medium treated with peptide/pDNA complexes at a charge ratio $=2$ contained 15.4 $\mu \mathrm{M}$ of the guanidino groups of these peptides. The R9 peptide had nine guanidino groups in one peptide, while (RRG)3 and (RRU)3 peptides had six guanidino groups. Therefore, the peptide (or 6-FAM) concentration of (RRG)3 and (RRU)3 peptides were 1.5-fold higher than that of the R9 peptide in the cell culture medium under the conditions of same guanidino group concentration. The uptake of (RRG)3 and (RRU)3 peptides by Huh-7 and HeLa cells was similar with the single administration of peptides and administration of peptide/pDNA complexes at all concentrations and charge ratios. 
The cellular uptake of R9 peptides added to peptide/pDNA complexes was significantly higher than that added to peptides alone under the conditions of high concentrations. Under the condition of the $61.5 \mu \mathrm{M}$ guanidino group (charge ratio $=8$ ) against Huh-7 cells, the uptake of R9 peptides in peptide/pDNA complexes was 16-fold higher than that of R9 peptides in peptides alone. R9 peptides added to peptides alone showed higher fluorescence intensities than those of (RRG)3 and (RRU)3 peptides at same guanidino concentrations in spite of higher dose of (RRG)3 and (RRU)3 peptides. The uptake of R9 peptides added to peptide/pDNA complexes at a charge ratio = 8 was 291and 36-fold higher than that of (RRG)3 and (RRU)3 peptides added to these complexes against Huh-7 cells, respectively, in which the transfection efficiency of R9/pDNA complexes was more than 1000-fold higher than those of (RRG)3 and (RRU)3/pDNA complexes (Figure 2). R9 peptides were internalized into cultured cells more efficiently than (RRG)3 and (RRU)3 peptides, especially in peptide/pDNA complexes, which may be the main reason for the higher transfection efficiency of R9/pDNA complexes. Figure 5 shows the results of a cytotoxiciy analysis performed under the same concentration of peptides as the cellular uptake experiments. The negligible cytotoxicities of all peptides with or without pDNA were detected in both Huh-7 and HeLa cells.

\section{3-4. Sizes and zeta-potentials of peptide/pDNA complexes}


The mean size and zeta-potential of peptide/pDNA complexes were markedly different according to the peptides (Table 1). R9/pDNA complexes at a charge ratio = 2 showed a size of $183 \mathrm{~nm}$ with a narrow PDI and positive charge of $+11.1 \mathrm{mV}$. Their sizes decreased to $88.2 \mathrm{~nm}$ and their zeta-potentials increased to $+18.2 \mathrm{mV}$ with an increase in the charge ratios. (RRG)3 and (RRU)3/pDNA complexes showed larger sizes of approximately 650-900 nm with a slightly wider PDI than R9/pDNA complexes. The zeta-potentials of (RRG)3 and (RRU)3/pDNA complexes were negative even at a charge ratio $=8$ in spite of an excess amount of cationic peptides being added $(-3.7 \mathrm{mV}$ for (RRG)3; -15.1 mV for (RRU)3). The introduction of neutral amino acid Gly and hydrophobic amino acid Aib into Arg-rich peptides may have influenced the formation of peptide/pDNA complexes.

\section{3-5. Fluorescence measurements}

The fluorescence intensities of peptide and peptide/pDNA complex solutions were measured in order to monitor the fluorescence quenching of fluorescent-labeled peptides and understand the physicochemical properties of each peptide/pDNA complex (Figure 6). Fluorescence quenching was only expected to occur with the formation of assembly structures through self-quenching $[14,15]$. The results of the peptide alone showed ideal curves in which the fluorescence intensities of (RRG)3 and (RRU)3 peptides were similar to and 1.5-fold higher than that of the R9 peptide at all concentrations. R9 and (RRG)3/pDNA complexes showed similar curves with negative maxima at a charge ratio 
$=1.25$, suggesting a stoichiometric charge ratio in these complexes. On the other hand, the curve of (RRU)3/pDNA complexes was markedly different from those of the R9 and (RRG)3/pDNA complexes and contained no distinct negative maximum. The fluorescence intensities of all peptide/pDNA complex solutions were similar at a charge ratio $=4$ (a guanidino group concentration: $410.3 \mu \mathrm{M})$, whereas those of (RRG)3 and (RRU)3 peptide solutions were approximately 1.5-fold higher than that of the R9 peptide solution at a guanidino group concentration of $410.3 \mu \mathrm{M}$. These results suggested the differences in the association behavior of each peptide with pDNA, which led to the different size and zeta-potential of each peptide/pDNA complex (Table 1).

\section{3-6. Effects of endocytosis inhibitors and low temperature on the internalization}

\section{of $\mathbf{R 9}$ peptides}

To clarify the mechanism underlying the internalization of R9 peptides and R9/pDNA complexes into Huh-7 cells, experiments on the inhibition of cellular uptake were performed using specific endocytosis inhibitors and a temperature of $4^{\circ} \mathrm{C}$ (Figure 7). The effects of the following endocytosis inhibitors on internalization were examined: amiloride (a specific inhibitor of macropinocytosis); chlorpromazine (a specific inhibitor of clathrin-mediated endocytosis); and nystatin (a specific inhibitor of caveolae-mediated endocytosis) [16-19]. Similar results were obtained from experiments using the R9 peptide alone and R9/pDNA complexes. Cellular uptake was significantly lowered by treatment with amiloride than by the treatment with no additive 
control, which implied that the internalization of R9 peptides added to peptides alone and peptide/pDNA complexes into Huh-7 cells mainly occurred via macropinocytosis. The low temperature $\left(4^{\circ} \mathrm{C}\right)$ incubation almost completely inhibited the internalization of R9 peptides, which suggested that R9 peptides and R9/pDNA complexes were internalized into Huh-7 cells through energy-dependent routes.

\section{3-7. Intracelluar distribution of $\mathrm{R9}$ peptides}

The intracellular distribution of R9 peptides (green) with or without pDNA (a guanidino group concentration: $61.5 \mu \mathrm{M}$; charge ratio $=8$ ) was investigated by CLSM against Huh-7 cells (Figure 8). LysoTracker Red (red) and Hoechst 33342 (blue) were used to label late endosomes/lysosomes and nuclei, respectively. The amount of peptides observed in the cells was markedly higher for peptide/pDNA complexes than peptides alone, which was consistent with the results obtained by the cellular uptake analysis (Figure 4). The colocalization of peptides with the late endosomes/lysosomes was quantified and shown in Figure 8B. More than 50\% of R9 peptides added to peptides alone were localized in late endosomes/lysosomes in contrast to only $33 \%$ of those added to peptide/pDNA complexes. These results revealed that R9 peptides added to peptide/pDNA complexes that were internalized into the Huh-7 cells achieved effective endosomal escape, which is one of the major obstacles for nonviral gene vectors. 


\section{Discussion}

An Arg-rich peptide is one of the most efficient CPPs, and therefore, has been developed as a tool for drug delivery systems (DDS) [1-10]. In the present study, an Arg nonapeptide (R9), which has frequently been utilized for DDS from the viewpoint of the balance between delivery efficiency and cytotoxicity, was evaluated for its pDNA transfection efficiency and cell-penetrating ability. Two additional types of nonapeptides, in which three Arg residues were replaced by the Gly or Aib residues, (RRG)3 and (RRU)3, respectively, were synthesized and evaluated to understand their effects on transfection and cell-penetrating abilities by replacing cationic amino acids (Arg) with neutral (Gly) and hydrophobic (Aib) amino acids (Figure 1).

Figure 2 showed the results of the transfection experiments using R9, (RRG)3, and (RRU)3/pDNA complexes with controls of naked pDNA and commercially available TurboFect/pDNA. In order to confirm cell type dependency on transfection efficiency of each peptide/pDNA complex, two types of cells, Huh-7 and HeLa cells, were used. The transfection efficiencies of (RRG)3 and (RRU)3/pDNA complexes were similar to that of naked pDNA against Huh-7 and HeLa cells. On the other hand, R9/pDNA complexes showed enhanced transfection efficiencies with an increase in charge ratios and comparable efficiencies to TurboFect/pDNA at a charge ratio $=8$ against Huh-7 cells. The replacement of Arg to Gly or Aib markedly reduced the transfection ability of Arg-rich peptides. Additional transfection experiments were carried out under conditions with prolonged post-incubation times (Figure 3). The transfection 
efficiency of R9/pDNA complexes did not change at any post-incubation time or in any cell line (Figures 3A and 3D). On the other hand, (RRG)3 and (RRU)3/pDNA complexes at a charge ratio $=4$ showed maximum transfection efficiencies after a 48 -h post-incubation and the transfection efficiency of (RRU)3/pDNA complexes at a charge ratio $=8$ was elevated after a 72-h post-incubation. The introduction of neutral or hydrophobic amino acids instead of Arg led to a delay in gene expression, which may have been due to an enhancement in stability through hydrophobic interactions and the slow release of pDNA from complexes as reported previously [20,21]. Another reason for delayed gene expression of (RRU)3/pDNA complexes might be that (RRU)3 peptides were stabilized against proteases in the serum compared to R9 and (RRG)3 peptides because of unnatural amino acids Aib introduced [22]. There was big difference of transfection efficiencies between Huh-7 and HeLa cells treated with (RRG)3 and (RRU)3/pDNA complexes. Transfection efficiency against HeLa cells is generally less than that against Huh-7 cells [21,23]; and therefore, we may not have been able to achieve sufficient transfection by (RRG)3 and (RRU)3/pDNA complexes against HeLa cells (Figures 3E and 3F). Arg oligopeptides composed of 4, 8, 12, or 16 Arg residues were previously reported to have low transfection efficiencies and the stearylation of these peptides increased their transfection efficiencies by approximately 100-fold [11]. In the present study, R9 peptides showed good transfection efficiencies similar to that of TuboFect under certain conditions, and thus, 6-FAM may function as a hydrophobic group not unlike a stearyl group. 
In order to elucidate the highest transfection efficiency of R9/pDNA complexes, the cellular uptake of peptides added to peptides alone and peptide/pDNA complexes was evaluated (Figure 4). In the experiments treated with peptides alone, R9 peptides showed the highest fluorescence intensity at all concentrations and against both cells. The amount of R9 peptides added to the cell culture medium was two-thirds those of (RRG)3 and (RRU)3 peptides. The cell-penetrating ability of R9 peptides was greater than those of (RRG)3 and (RRU)3 peptides. The amounts of (RRG)3 and (RRU)3 peptides were almost the same between the peptides alone and peptide/pDNA complexes, while the uptake of R9 peptides was markedly higher with peptide/pDNA complexes than with peptides alone at high concentrations (charge ratios). These results may be explained by the different formation and physicochemical properties of peptide/pDNA complexes. The fluorescence intensity of (RRU)3/pDNA complex solutions indicated no negative maximum at any charge ratio, which was quite different from those of the R9 and (RRG)3/pDNA complexes (Figure 6). (RRU)3 peptides added to pDNA solution were consistently less capable of associating with pDNA than R9 peptides, which may have led to highly negative zeta-potentials even at a charge ratio $=8$ (Table 1). The fluorescence intensity of (RRG)3/pDNA complexes at a charge ratio $=4$ was similar to that of R9/pDNA complexes, although the concentration of RRG peptides was 1.5-fold higher than that of R9 peptides. The zeta-potential of (RRG)3/pDNA complexes was negative even with an excess amount of peptides and their size was approximately $800 \mathrm{~nm}$, while R9/pDNA complexes at a charge ratio $=8$ showed a 
zeta-potential of $+18.2 \mathrm{mV}$ and size of $88.2 \mathrm{~nm}$. The formation of R9/pDNA complexes with highly cationic surfaces and sized less than $100 \mathrm{~nm}$ may contribute to their more efficient cellular uptake than R9 peptides alone. Taken together with these results, decreases in cationic amino acids and increases in hydrophobic amino acids in Arg-rich peptides greatly affected the formation and physicochemical properties of peptide/pDNA complexes, resulting in reductions in cellular uptake and transfection efficiency. R9 peptides, regardless of being formed with or without pDNA, were taken up by Huh-7 cells through the same mechanism, macropinocytosis, which was consistent with previous paper that macropinocytosis played a major role in Arg-rich peptides internalization (Figure 7) [24]. However, amiloride inhibited approximately 65\% of R9 peptide internalization for R9/pDNA complexes and 45\% for R9 peptides alone. These differences may have contributed to the significant differences observed in the colocalization ratio with the late endosomes/lysosomes (Figure 8B) because macropinosomes are thought to be difficult to fuse into lysosomes and inherently leaky vesicles $[16,25]$.

\section{Conclusion}

We evaluated the transfection efficiencies and cell-penetrating abilities of three Arg-rich peptides: R9, (RRG)3, and (RRU)3. The replacement of cationic Arg in the Arg-rich peptides with neutral Gly and hydrophobic Aib markedly decreased the cell-penetrating abilities of and altered the physicochemical properties of peptide/pDNA 
complexes, which resulted in marked reductions in pDNA transfection abilities. Based on these results, novel Arg-rich CPPs may be designed and developed for pDNA delivery.

\section{Acknowledgements}

This work was financially supported in part by JSPS KAKENHI Grant Number 25713008 (MO) and 26460169 (YD), by the Kurata Grant awarded by the Kurata Memorial Hitachi Science and Technology Foundation (MO), and scholarship support from the Shoshisha Foundation (HY).

\section{References}

[1] Nagahara, H.; Vocero-Akabani, A. M.; Snyder, E. L.; Ho, A.; Latham, D. G.; Lissy, N. A.; Becker-Hapak, M.; Ezhevsky, S. A.; Dowdy, S. F. Nat. Med. 1998, 4, 1449.

[2] Nakase, I.; Takeuchi, T.; Tanaka, G.; Futaki, S. Adv. Drug Deliv. Rev. 2008, 60, 598.

[3] Schmidt, N.; Mishra, A.; Lai, G. H.; Wong, G. C. L. FEBS Lett. 2010, 584, 1806.

[4] Umezawa, N.; Gelman, M. A.; Haigis, M. C.; Raines, R. T.; Gellman, S. H. J. Am. Chem. Soc. 2002, 124, 368.

[5] Wender, P. A.; Gralliher, W. C.; Goun, E. A.; Jones, L. R.; Pillow, T. H. Adv. Drug Deliv. Rev. 2008, 60, 452. 
[6] El-Sayed, A.; Futaki, S.; Harashima, H. AAPS J. 2008, 11, 13.

[7] Kato, T.; Oba, M.; Nishida, K.; Tanaka, M. Bioconjugate Chem. 2014, 25, 1761.

[8] Veldhoen, S.; Laufer, S. D.; Restle, T. Int. J. Mol. Sci. 2008, 9, 1276.

[9] Hoyer, J.; Neundorf, I. Acc. Chem. Res. 2012, 45, 1048.

[10] Nakase, I.; Akita, H.; Kogure, K.; Gräslund, A.; Langel, Ü.; Harashima, H.; Futaki, S. Acc. Chem. Res. 2012, 45, 1132.

[11] Futaki, S.; Ohashi, W.; Suzuki, T.; Niwa, M.; Tanaka, S.; Ueda, K.; Harashima, H.; Sugiura, Y. Bioconjugate Chem. 2001, 12, 1005.

[12] Yamashita, H.; Demizu, Y.; Shoda, T.; Sato, Y.; Oba, M.; Tanaka, M.; Kurihara, M. Bioorg. Med. Chem. 2014, 22, 2403.

[13] Ge, Z.; Chen, Q.; Osada, K.; Liu, X.; Tockary, T. A.; Uchida, S.; Dirisala, A.; Ishii, T.; Nomoto, T.; Toh, K.; Matsumoto, Y.; Oba, M.; Kano, M. R.; Itaka, K.; Kataoka, K. Biomaterials 2014, 35, 3416.

[14] Gruber, H. J.; Hahn, C. D.; Kada, G.; Riener, C. K.; Harms, G. S.; Ahrer, W.; Dax, T. G.; Knaus, H. G. Bioconjugate Chem. 2000, 11, 696. 
[15] Christie, R. J.; Miyata, K.; Matsumoto, Y.; Nomoto, T.; Menasco, D.; Lai, T. C.; Pennisi, M.; Osada, K.; Fukushima, S.; Nishiyama, N.; Yamasaki, Y.; Kataoka, K. Biomacromolecules 2011, 12, 3174.

[16] Khalil, I. A.; Kogure, K.; Akita, H.; Harashima, H. Pharmacol. Rev. 2006, 58, 32.

[17] von Gersdorff, K.; Sanders, N. N.; Vandenbroucke, R.; De Smedt, S. C.; Wagner, E.; Ogris, M. Mol. Ther. 2006, 14, 745.

[18] Oba, M.; Tanaka, M. Biol. Pharm. Bull. 2012, 35, 1064.

[19] Kato, T.; Tanaka, M.; Oba, M. PLoS ONE 2013, 8, e82876.

[20] Oba, M.; Aoyagi, K.; Miyata, K.; Matsumoto, Y.; Itaka, K.; Nishiyama, N.; Yamasaki, Y.; Koyama, H.; Kataoka, K. Mol. Pharmaceutics 2008, 5, 1080.

[21] Oba, M.; Miyata, K.; Osada, K.; Christie, R. J.; Sanjoh, M.; Li, W.; Fukushima, S.; Ishii, T.; Kano, M. R.; Nishiyama, N.; Koyama, H.; Kataoka, K. Biomaterials 2011, 32. 652.

[22] Wada, S.; Tsuda, H.; Okada, T.; Urata, H. Bioorg. Med. Chem. Lett. 2011, 21, 5688.

[23] Nidome, T.; Takaji, K.; Urakawa, M.; Ohmori, N.; Wada, A.; Hirayama, T.; Aoyagi, H. Bioconjugate Chem. 1999, 10, 773. 
[24] Nakase, I.; Niwa, M.; Takeuchi, T.; Sonomura, K.; Kawabata, N.; Koike, Y.;

Takehashi, M.; Tanaka, S.; Ueda, K.; Simpson, J. C.; Jones, A. T.; Sugiura, Y.; Futaki, S. Mol. Ther. 2004, 10, 1011.

[25] Mayor, S.; Pagano, R. E. Nat. Rev. Mol. Cell Biol. 2007, 8, 603. 


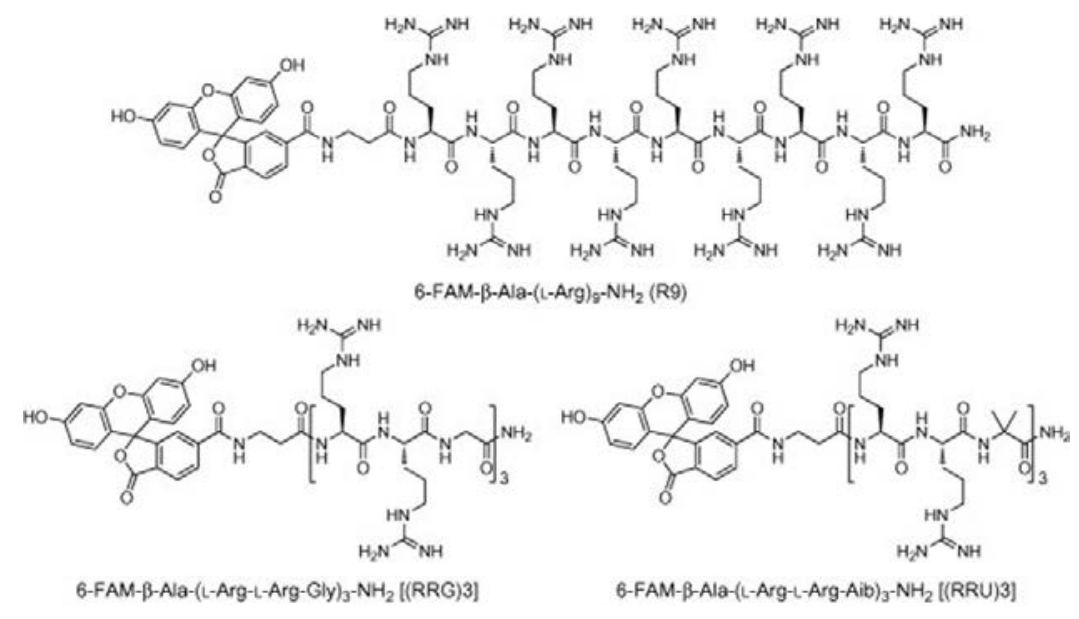

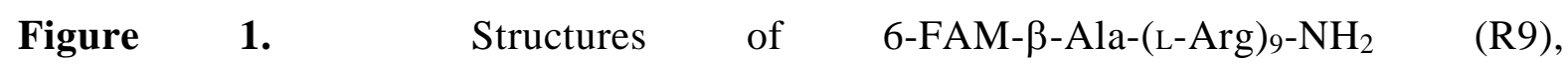
6-FAM- $\beta$-Ala-(L-Arg-L-Arg-Gly) $)_{3}-\mathrm{NH}_{2} \quad$ [(RRG)3], and 6-FAM- $\beta$-Ala-(L-Arg-L-Arg-Aib) ${ }_{3}-\mathrm{NH}_{2}$ [(RRU)3]. 

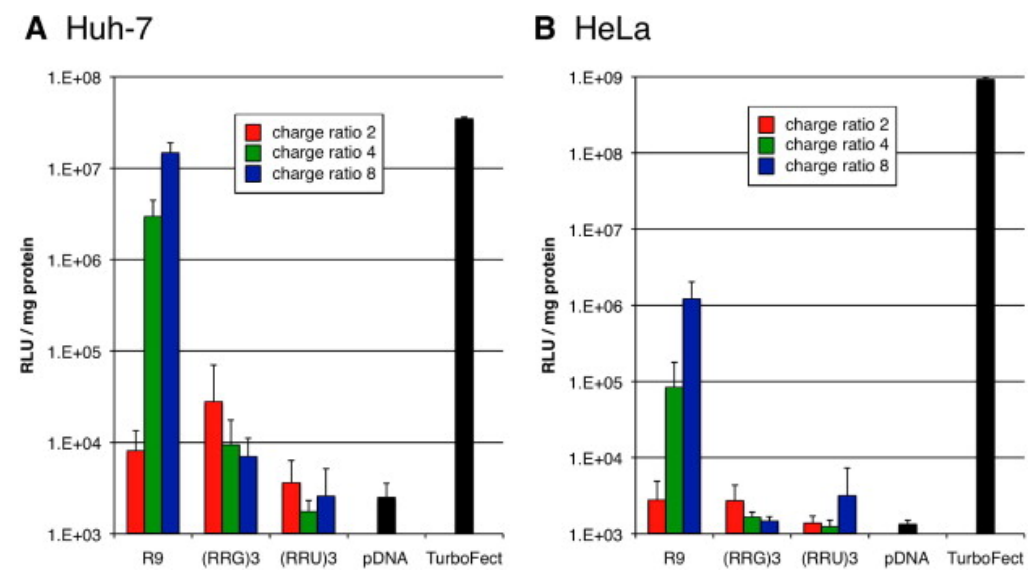

Figure 2. Transfection efficiencies of peptide/pDNA complexes prepared at various charge ratios, naked pDNA, and commercially available TurboFect/pDNA against Huh-7 cells (A) and HeLa cells (B). Error bars represent the standard deviation, $n=4$. 

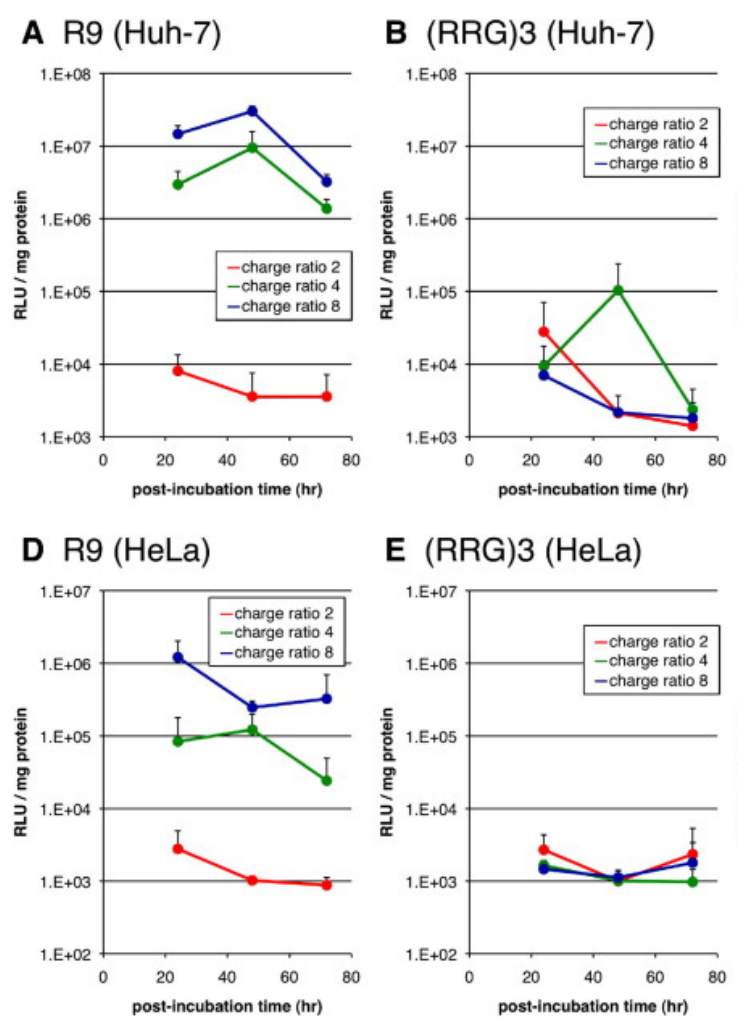
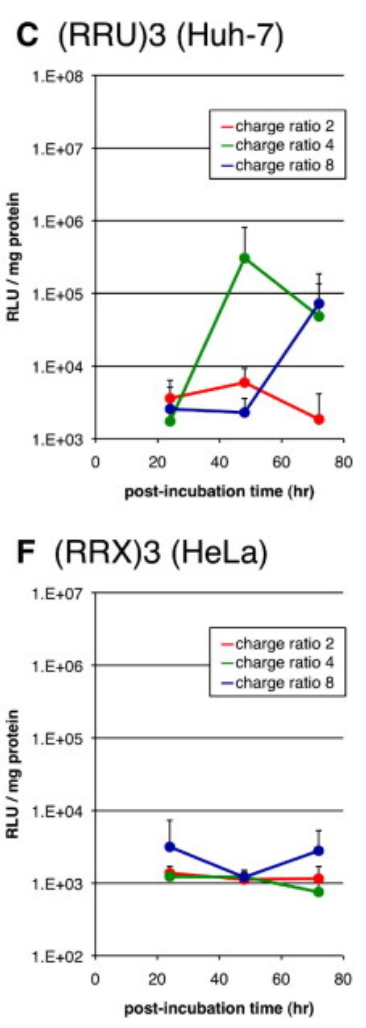

Figure 3. Post-incubation time dependencies of transfection efficiencies of R9 (A, D), (RRG)3 (B, E), and (RRU)3/pDNA complexes (C, F) against Huh-7 cells (A-C) and HeLa cells (D-F). Error bars represent the standard deviation, $n=4$. 

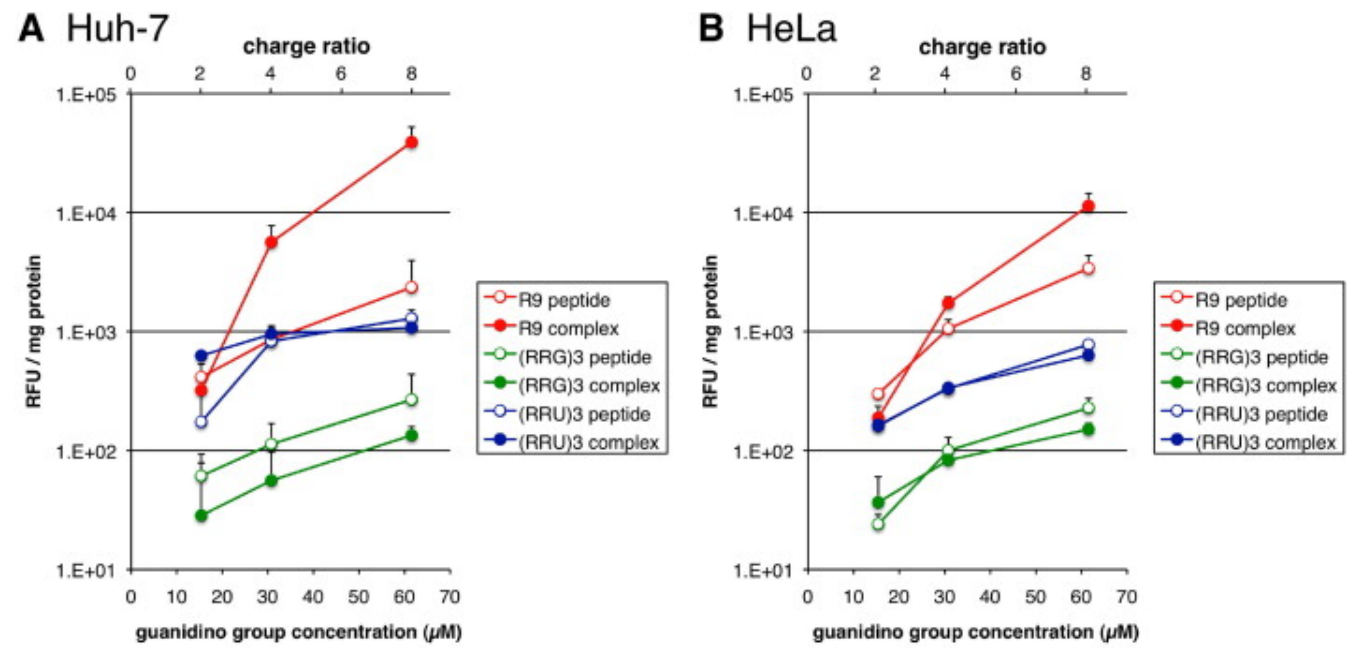

Figure 4. Cellular uptake of peptides and peptide/pDNA complexes at various peptide concentrations and charge ratios against Huh-7 cells (A) and HeLa cells (B). Error bars represent the standard deviation, $\mathrm{n}=4$. 
A Huh-7

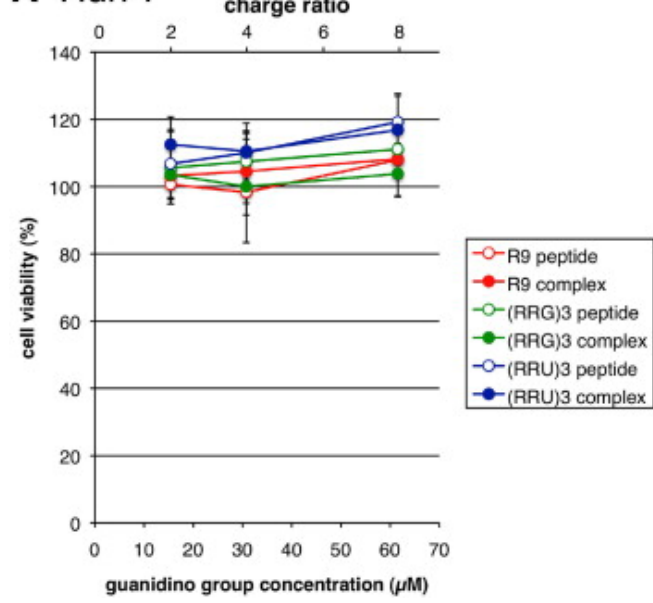

B HeLa charge ratio

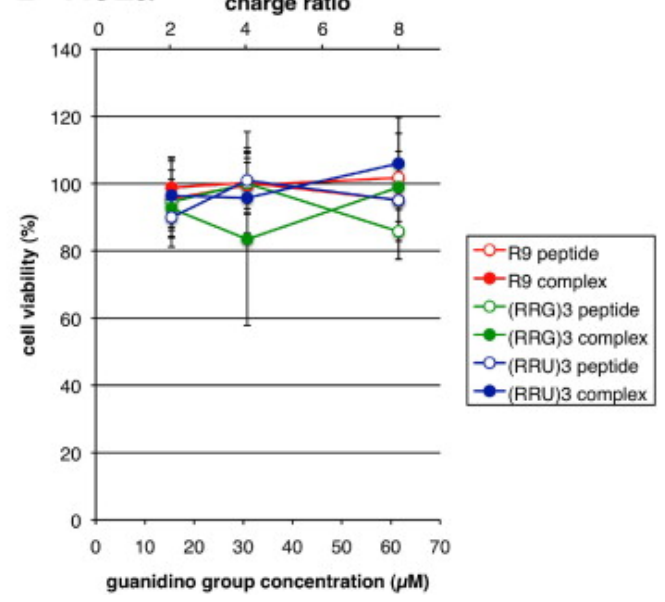

Figure 5. Cytotoxicities of peptides and peptide/pDNA complexes at various peptide concentrations and charge ratios against Huh-7 cells (A) and HeLa cells (B). Error bars represent the standard deviation, $\mathrm{n}=6$. 


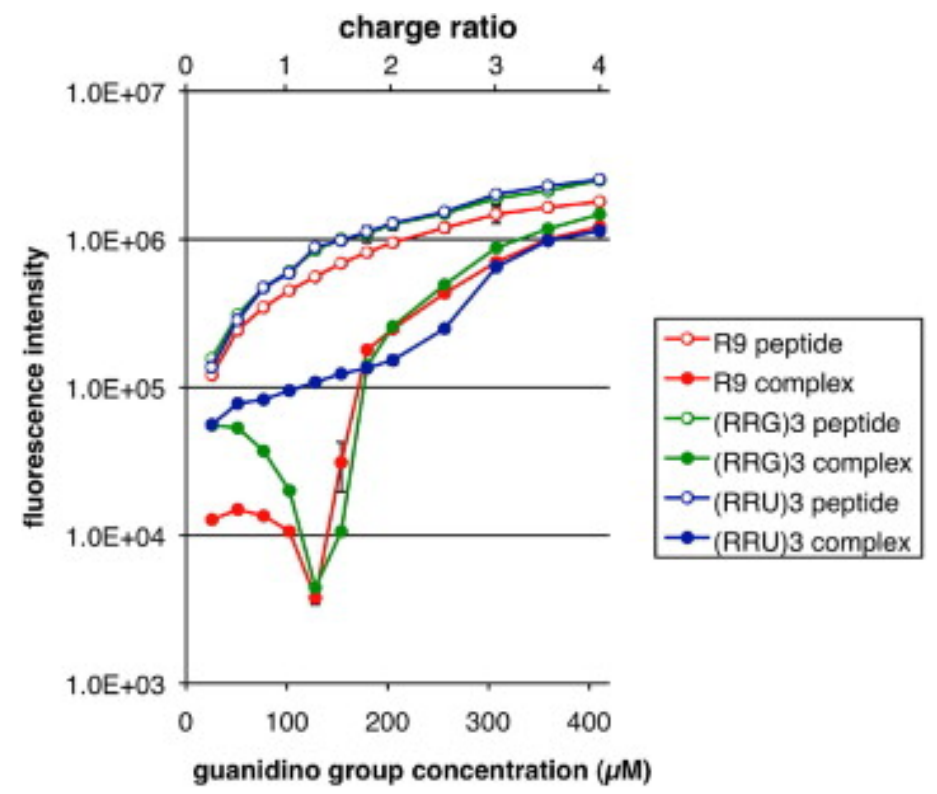

Figure 6. Fluorescence intensities of peptide and peptide/pDNA complex solutions at various peptide concentrations and charge ratios. Error bars represent the standard deviation, $\mathrm{n}=3$. 


\section{A R9 peptide}

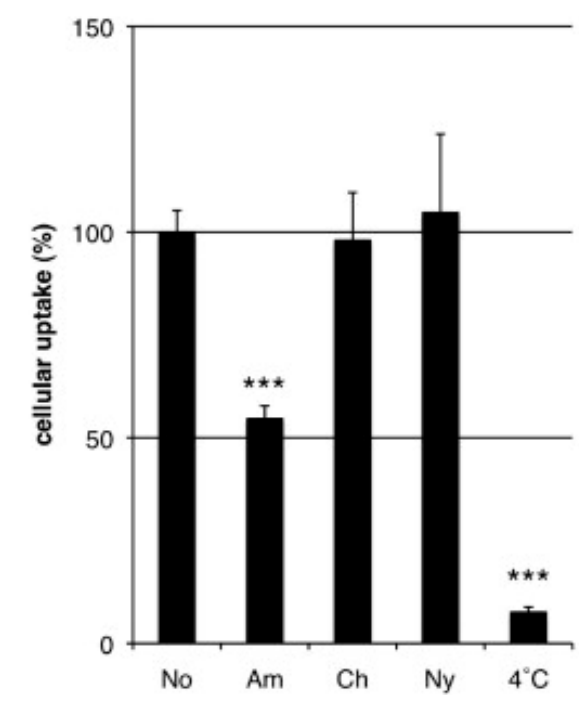

B R9 complex

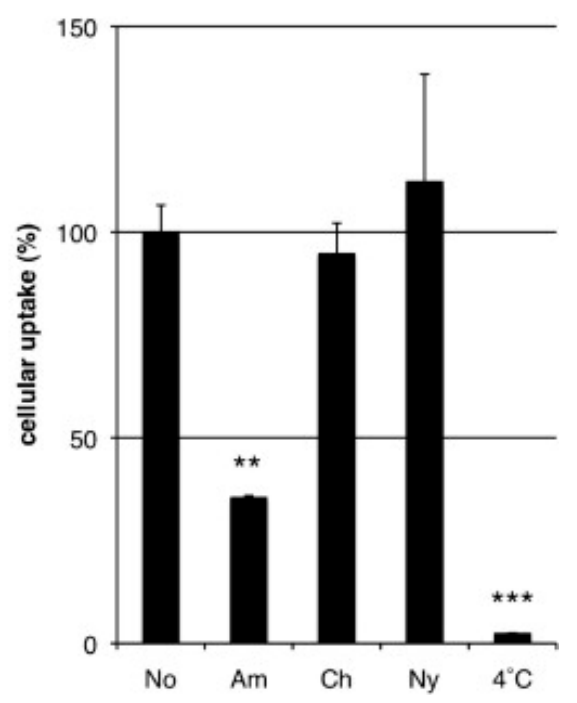

Figure 7. Effects of inhibitors (No: no additive; amiloride (Am): a macropinocytosis inhibitor; chlorpromazine (Ch): a clathrin-mediated endocytosis inhibitor; nystatin (Ny): a caveolae-mediated endocytosis inhibitor) and low temperature $\left(4^{\circ} \mathrm{C}\right)$ on the internalization of R9 peptides (A) and R9/pDNA complexes (B) against Huh-7 cells. Error bars represent the standard deviation, $\mathrm{n}=4 .{ }^{* *} P<0.01$ and ${ }^{* *} P<0.001$. 


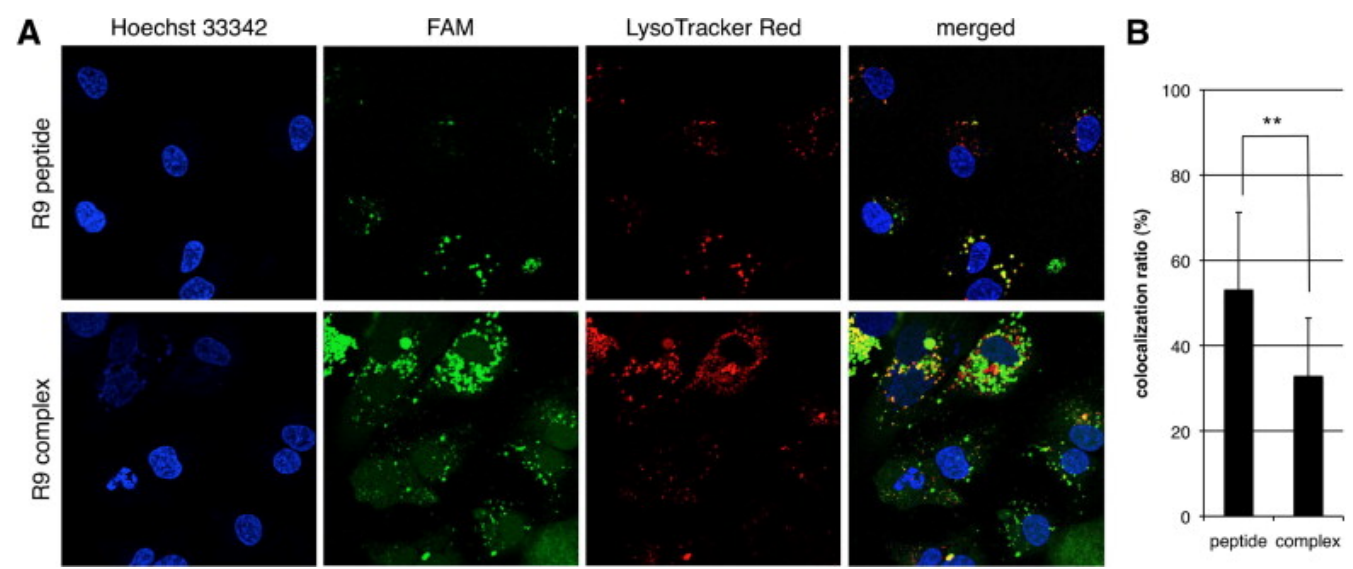

Figure 8. (A) CLSM observations of the intracellular distribution of R9 peptides and R9 peptide/pDNA complexes (green) with late endosomes/lysosomes (red) and nuclei (blue) stained using LysoTracker Red and Hoechst 33342, respectively. (B) Quantification of peptides colocalized with LysoTracker Red in Huh-7 cells. Error bars represent the standard deviation, $\mathrm{n}=16 .{ }^{* *} \mathrm{P}<0.01$. 
Table 1. Zeta-potential and size of each peptide/pDNA complex prepared at various charge ratios.

\begin{tabular}{|c|c|c|c|c|}
\hline & charge ratio & zeta-potential (mV) & size (nm) & $\operatorname{PDI}\left(\mu / \Gamma^{2}\right)$ \\
\hline \multirow[t]{3}{*}{ R9 } & 2 & $+11.1 \pm 0.29$ & $183 \pm 12.4$ & $0.025 \pm 0.006$ \\
\hline & 4 & $+13.8 \pm 0.06$ & $105 \pm 1.3$ & $0.026 \pm 0.003$ \\
\hline & 8 & $+18.2 \pm 0.06$ & $88.2 \pm 0.7$ & $0.040 \pm 0.018$ \\
\hline \multirow[t]{3}{*}{$(\mathrm{RRG}) 3$} & 2 & $-21.3 \pm 0.23$ & $668 \pm 22.1$ & $0.092 \pm 0.118$ \\
\hline & 4 & $-4.7 \pm 0.23$ & $718 \pm 75.7$ & $0.158 \pm 0.044$ \\
\hline & 8 & $-3.7 \pm 0.40$ & $767 \pm 21.1$ & $0.404 \pm 0.273$ \\
\hline \multirow[t]{3}{*}{$(\mathrm{RRU}) 3$} & 2 & $-42.6 \pm 0.17$ & $80 \pm 0.2$ & $0.109 \pm 0.016$ \\
\hline & 4 & $-14.7 \pm 0.25$ & $834 \pm 23.7$ & $0.036 \pm 0.028$ \\
\hline & 8 & $-15.1 \pm 0.35$ & $876 \pm 59.3$ & $0.164 \pm 0.030$ \\
\hline
\end{tabular}

